\title{
Mutational landscape of gastric cancer and clinical application of genomic profiling based on target next-generation sequencing
}

Hui Cai ${ }^{1 \dagger}$, Changqing Jing ${ }^{2+}$, Xusheng Chang ${ }^{1}$, Dan Ding ${ }^{1}$, Ting Han ${ }^{1}$, Junchi Yang ${ }^{1}$, Zhengmao Lu',

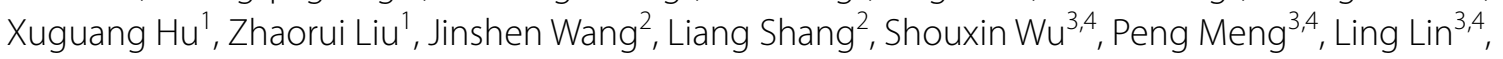
Jiangman Zhao ${ }^{3,4^{*}}$, Mingming Nie ${ }^{1^{*}}$ and Kai Yin ${ }^{1 *}$ (D)

\begin{abstract}
Background: Gastric cancer (GC) is a leading cause of cancer deaths, and an increased number of GC patients adopt to next-generation sequencing (NGS) to identify tumor genomic alterations for precision medicine.

Methods: In this study, we established a hybridization capture-based NGS panel including 612 cancer-associated genes, and collected sequencing data of tumors and matched bloods from 153 gastric cancer patients. We performed comprehensive analysis of these sequencing and clinical data.

Results: 35 significantly mutated genes were identified such as TP53, AKAP9, DRD2, PTEN, CDH1, LRP2 et al. Among them, 29 genes were novel significantly mutated genes compared with TCGA study. TP53 is the top frequently mutated gene, and tends to mutate in male $(p=0.025)$ patients and patients whose tumor located in cardia $(p=0.011)$. High tumor mutation burden (TMB) gathered in TP53 wild-type tumors $(p=0.045)$. TMB was also significantly associated with DNA damage repair (DDR) genes genotype $(p=0.047)$, Lauren classification $(p=1.5 e-5)$, differentiation (1.9e-7), and HER2 status $(p=0.023) .38 .31 \%$ of gastric cancer patients harbored at least one actionable alteration according to OncoKB database.
\end{abstract}

Conclusions: We drew a comprehensive mutational landscape of 153 gastric tumors and demonstrated utility of target next-generation sequencing to guide clinical management.

Keywords: Gastric cancer, Next-generation sequencing, Tumor mutation burden, Clinical actionable alterations

\section{Background}

Gastric cancer (GC) is the third leading cause of cancerrelated mortality in the world, with the highest incidence occurring in Eastern Asia (particularly in China) and lowest in Northern America [1]. In China, there were 679,100 new cases and 498,000 deaths of gastric cancer in

\footnotetext{
*Correspondence: zhaojiangman86@163.com; niemm@smmu.edu.cn; kyin67@126.com

${ }^{\dagger}$ Hui Cai and Changqing Jing contributed equally to this work

${ }^{1}$ Department of Gastrointestinal Surgery, Changhai Hospital, The Second Military Medical University, 168 Changhai Road, Yangpu District, Shanghai 200433, China

${ }^{3}$ Zhangjiang Center for Translational Medicine, Shanghai Biotecan Pharmaceuticals Co., Ltd., 180 Zhangheng Road, Shanghai 201204, China Full list of author information is available at the end of the article
}

2015, which account for above half of the world's gastric cancer deaths [2]. Gastric cancer is a heterogeneous disease with phenotypic diversity that encompasses various molecular subtypes, which can be subdivided into intestinal and diffuse types according to the Lauren classification [3]. Recent studies using next-generation sequencing (NGS) have revealed an extensive repertoire of potential cancer-driving genes and drew mutational landscape of gastric cancer. The Cancer Genome Atlas (TCGA) project divided gastric cancer into four subtypes: EpsteinBarr virus (EBV) positive, microsatellite instability (MSI), genomically stable (GS) and chromosomal instability (CIN) [4]. Chen et al. performed whole-exome sequencing (WES) on paired normal-cancer tissues of 78 gastric 
cancer patients in northern China (Tianjin), which distinguished two GC subtypes with either high-clonality (HiC) or low-clonality (LoC) [5].

Over the last decade, NGS has enabled application of clinical genomics to the diagnosis and treatment of cancers. Taking lung cancer as an example, it has become standard practice to profile tumors for driver mutations. Target capture sequencing may identify actionable mutated driver genes and further determine tumor mutation burden (TMB), which is more cost effective and efficient, and achieves higher sequencing depth than WES. Zehir et al. have reported the mutational landscape of 10,000 pan-cancer patients using a hybridization capture-based NGS panel (MSK-IMPACT) [6]. In recent years, a number of studies about gastric cancer adopted target next-generation sequencing technology [7-9].

We developed a hybridization capture-based NGS panel, Biotecan PanCancer Panoramic Detection (BTCPCPD), which is capable of detecting protein-coding mutations, copy number alterations (CNAs), TMB and selected promoter mutations in 612 cancer-associated genes $(2.75 \mathrm{M})$ (Additional file 1). BTC-PCPD panel was designed referring to cancer-related database, clinical guidelines, and high-quality references, which included cancer genetic risk genes, targeted drugs (approved by FDA, clinical trials) and chemotherapy associated genes, and prognosis genes (Additional file 1). In this study, we performed BTC-PCPD panel sequencing on tumors and paired peripheral blood from 153 patients with gastric tumor. Using these data, we drew a comprehensive mutational landscape of 153 gastric tumors and demonstrated utility of massively parallel DNA sequencing of tumors to guide clinical management.

\section{Methods}

\section{Patients and samples}

We recruited 153 gastric cancer patients from Department of Gastrointestinal Surgery in Changhai Hospital (Shanghai, China) and Department of Gastrointestinal Surgery in Shandong Provincial Hospital (Jinan, China) between February 2017 and November 2018. 134 fresh frozen tumor tissues and 19 Formalin-Fixed and ParaffinEmbedded (FFPE) tumor tissues were collected after surgery. We used germline DNA from blood as a reference for detecting somatic alterations. Clinical characteristics of 153 gastric cancer patients were showed in Table 1.

\section{DNA extraction and quality control}

gDNA from fresh tissue was extracted by QIAamp DNA Mini Kit, gDNA from blood by QIAamp DNA Blood Mini Kit, gDNA from FFPE tissues by GeneRead DNA FFPE Kit (Qiagen, Hilden, Germany). Quantity and purity of gDNA were assessed by Qubit ${ }^{\circledR} 3.0$
Table 1 Clinical characteristics of patients enrolled in this study according to TP53 status

\begin{tabular}{|c|c|c|c|c|}
\hline \multirow{2}{*}{$\begin{array}{l}\text { Clinical } \\
\text { characteristics }\end{array}$} & \multirow[t]{2}{*}{ No. of patients } & \multicolumn{2}{|l|}{ TP53 } & \multirow[t]{2}{*}{$p$ value } \\
\hline & & Wild type & Mutated & \\
\hline Total & 153 & $63(41.18 \%)$ & $90(58.82 \%)$ & \\
\hline \multicolumn{5}{|l|}{ Gender } \\
\hline Male & 103 & 36 & 67 & $0.025^{*}$ \\
\hline Female & 50 & 27 & 23 & \\
\hline $\begin{array}{l}\text { Age median } \\
\text { (range) }\end{array}$ & $59(19-80)$ & & & \\
\hline \multicolumn{5}{|l|}{ Stage } \\
\hline । & 25 & 12 & 13 & 0.206 \\
\hline ॥ & 39 & 18 & 21 & \\
\hline III & 69 & 22 & 47 & \\
\hline \multicolumn{5}{|c|}{ Lauren classification } \\
\hline Diffuse & 50 & 23 & 27 & 0.163 \\
\hline Intestinal & 47 & 13 & 34 & \\
\hline Mixed & 24 & 10 & 14 & \\
\hline \multicolumn{5}{|l|}{ Differentiation } \\
\hline Poorly & 62 & 29 & 33 & 0.21 \\
\hline $\begin{array}{l}\text { Moderately and } \\
\text { poorly }\end{array}$ & 27 & 9 & 18 & \\
\hline Moderately & 45 & 14 & 31 & \\
\hline \multicolumn{5}{|l|}{ Location } \\
\hline Cardia & 24 & 4 & 20 & $0.011^{*}$ \\
\hline $\begin{array}{l}\text { Gastric (fundus, } \\
\text { body, antrum, } \\
\text { pylorus) }\end{array}$ & 122 & 56 & 66 & \\
\hline \multicolumn{5}{|c|}{ Lymphatic metastasis } \\
\hline Yes & 89 & 31 & 58 & 0.067 \\
\hline No & 47 & 24 & 23 & \\
\hline \multicolumn{5}{|l|}{ Drinking } \\
\hline Yes & 21 & 6 & 15 & 0.228 \\
\hline No & 127 & 54 & 73 & \\
\hline \multicolumn{5}{|l|}{ Smoke } \\
\hline Yes & 30 & 10 & 20 & 0.368 \\
\hline No & 118 & 50 & 68 & \\
\hline \multicolumn{5}{|l|}{ HER-2 IHC } \\
\hline Positive & 11 & 2 & 9 & 0.208 \\
\hline Negative & 110 & 43 & 67 & \\
\hline
\end{tabular}

Patients were not showed whose clinical data was missing

${ }^{*} \mathrm{p}<0.05,{ }^{* *} \mathrm{p}<0.01,{ }^{* * *} \mathrm{p}<0.001$

Fluorometer (Invitrogen, Carlsbad, CA, USA) and NanoDrop ND-1000 (Thermo Scientific, Wilmington, DE, USA). Fragmentation status were evaluated by the Agilent 2200 TapeStation system using the Genomic DNA ScreenTape assay (Agilent Technologies, Santa Clara, CA, USA) able to produce a DNA Integrity Number (DIN). An additional quality control (QC) step to assess FFPE DNA integrity was performed using a multiplex Polymerase Chain Reaction (PCR) approach [10]. Briefly, 
30 ng of gDNA were amplified using three different-size set of primers of glyceraldehyde-3-phosphate dehydrogenase (GAPDH) gene (200-300-400 base pair), and the concentration of PCR products was determined by Agilent 2100 Bioanalyzer instrument (Agilent Technologies). Then, to estimate FFPE gDNA fragmentation, we evaluated an Average Yield Ratio (AYR) value, calculated by yield ratio of each amplicon compared with a reference DNA (Promega Madison, WI, USA).

\section{Library preparation and hybridization capture}

A total of 300 ng of each gDNA sample based on Qubit quantification were mechanically fragmented on a E220 focused ultrasonicator Covaris (Covaris, Woburn, MA, USA). Two hundred nanogram of sheared gDNA were used to perform end repair, A-tailing and adapter ligation with either Agilent SureSelect XT (Agilent Technologies) or KAPA library preparation kits (KapaBiosystems Inc. Wilmington, MA, USA), following the manufacturer instructions. Subsequently, the libraries were captured using Agilent SureSelect XT custom 0.5-2.9 M (Agilent Technologies) probes, and finally amplified.

\section{Clustering and sequencing}

After QC and quantification by Agilent 2100 Bioanalyzer (Agilent Technologies) and Qubit ${ }^{\circledR}$ 2.0 Fluorometer (Invitrogen), the libraries were sequenced on an Illumina Next 500 platform (IlluminaInc, San Diego, CA, USA) High Output mode, $2 \times 75$ cycles.

\section{Bioinformatics analysis}

Clean data was obtained following filtering adapter, low quality reads and reads with proportion of $\mathrm{N}>10 \%$. Reads were aligned to the reference human genome (UCSC hg19) using the Burrows-Wheeler Aligner v. 0.7.12. Next, the Picard and Genome Analysis Toolkit (GATK v.3.2) method was adopted for duplicate removal, local realignment and Base Quality Score Recalibration, and generated the quality statistics, including mapped reads, mean mapping quality and mean coverage. Finally, the GATK HaplotypeCaller was used for SNV and InDel identification.

Variants were annotated using the ANNOVAR software tool. Annotations for mutation function (including frameshift insertion/deletion, non-frameshift insertion/deletion, synonymous SNV, nonsynonymous SNV, stop gain stop loss), mutation location [such as exonic, intronic, splicing, upstream, downstream, $3^{\prime}$ untranslated region (UTR), $5^{\prime}$ UTR and so on], amino acid changes, 1000 Genomes Project data and dbSNP reference number were performed.

Somatic SNVs and InDels of tumors compared with matched normal tissue were named and functionally annotated using MuTect v. 1.1.4 and Varscan2 v. 2.3.9 software. The mutations with variant allele frequency $>5 \%$ were defined as high confidence mutations. Tumor mutation burden (TMB) was defined as the number of all somatic base substitution and indel per mega base excluding synonymous mutation.

MutSigCV v.0.9 [11] was used to identify significantly mutated genes $(\mathrm{q}<0.1)$. Then, gene mutation data were downloaded from TCGA database, and comparative analysis was performed using the sequencing data produced in the present study. Varscan2 software was used for identifying and annotating CNAs (|log2_ratio $\mid>1)$. To account for differences in sequence data input, the ratio of tumor depth to normal depth was normalized with the data-ratio parameter. The relative copy number in tumor in computed from the log-base- 2 of the ratio of tumor depth to normal depth.

\section{Statistical analysis}

The mutation landscape across a cohort, including SNVs, InDels and mutational burden, were created by Genomic Visualizations in R (GenVisR). The custom mutation lists of proteins were visualized by MutationMapper tool from cBioPortal. Gene ontology (GO) and Kyoto Encyclopedia of Genes and Genomes (KEGG) enrichment analysis were performed to investigate the biological importance of the somatic mutated genes of all samples using the ClusterProfiler in R software [12]. The cutoff of p-values $<0.05$ and FDR $<0.05$ were used to assess the significance of enrichment terms. The nonparametric Mann-Whitney $U$ test was subsequently used to test for significance in difference of means between two populations.

\section{Results}

Characteristics of patients and sequencing data

Clinical characteristics of 153 gastric cancer patients were showed in Table 1. Primary tumors were from the following anatomic locations of the stomach: 24 from cardia, 64 from the antrum, 36 from the body, 4 from the pylorus, 3 from the fundus, and 15 across over two locations. All histopathologic diagnoses were subjected to independent reviews by at least two senior pathologists. According to Lauren classification, 50 cases were classified as diffuse-type, 47 as intestinal-type, and 24 as mixed-type. Among these patients, 25 were stage I, 39 were stage II, and 69 were stage III.

Massively parallel DNA sequencing achieved an average of $594 \times$ coverage of the tumor genomes with 0.91 of $\mathrm{Q} 30$, and $142 \times$ coverage of the germline genomes with 0.91 of Q30. There was no significant difference of sequencing depth and Q30 between fresh tissues and FFPE tissues, which showed FFPE tissues obtained good sequence data. 


\section{Landscape of somatic mutations}

We used MutSigCV tool to define significantly mutated genes in 153 gastric tumors, and identified 35 significantly mutated genes (Fig. 1a). The five most frequently mutated genes were TP53 (59.09\%), DRD2 (14.29\%), CDH1 (13.64\%), AKAP9 (14.93\%) and ATM (11.69\%) (Fig. 1a). Lollipop plots showed the type and location of all significant gene mutations (Additional file 2). Obviously, the BTC-PCPD panel results were highly consistent with the TCGA finding, exhibiting strong concordance of the 35 mutated genes and population frequencies of mutations detected (Fig. 1b), but relatively weaker concordance of all somatic mutated genes (Additional file 3). But there were still some deflected genes such as APC, LRP2, DRD2 (Fig. 1b). TP53 was the most frequently mutated gene in TCGA $(47.59 \%)$ [4] and BTC-PCPD cohort (59.09\%). We found TP53 tended to mutate in male $(\mathrm{p}=0.025)$ gastric cancer

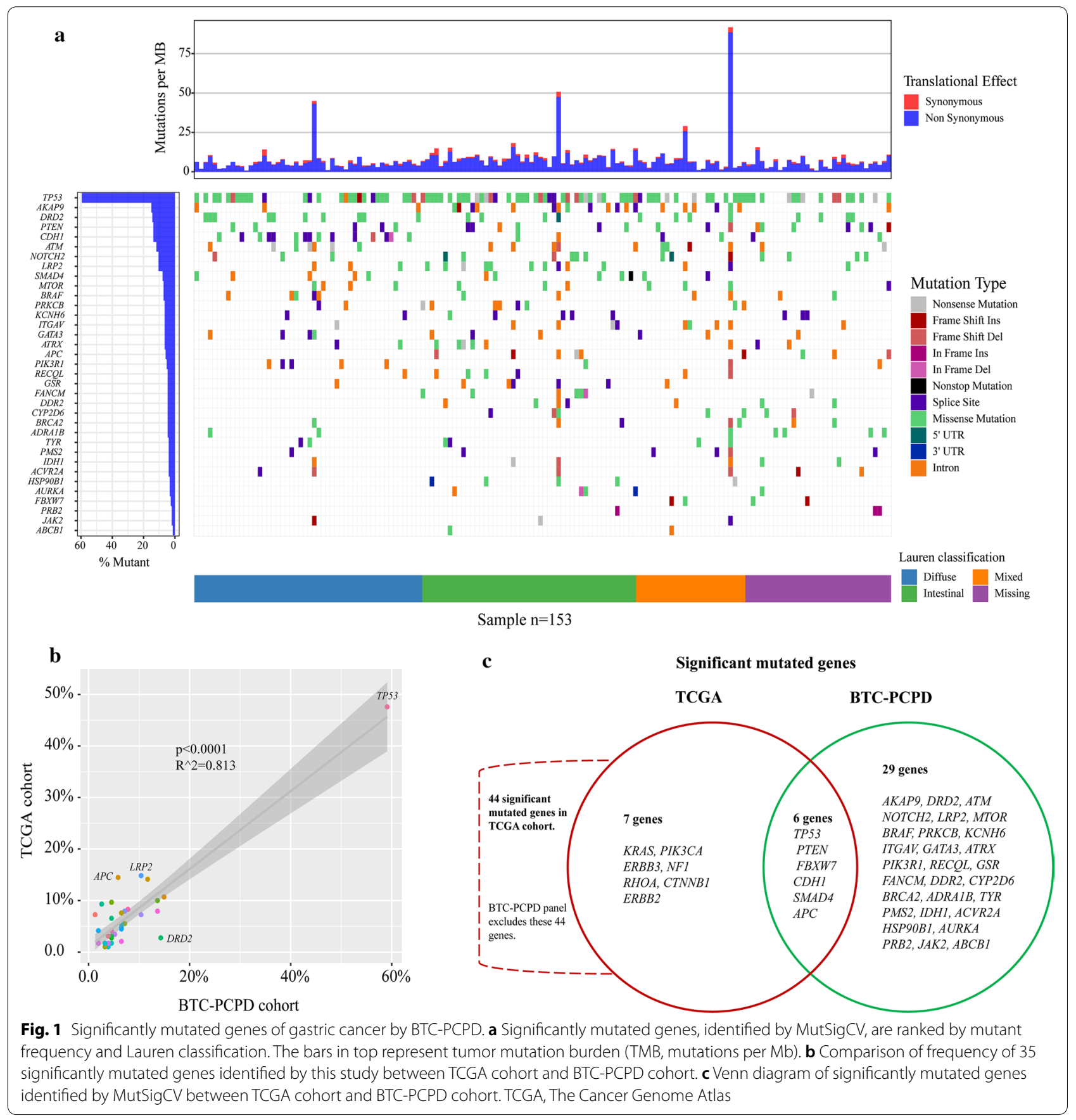


patients and patients whose tumor located in cardia $(\mathrm{p}=0.011)($ Table 1$)$.

Comparative analysis of significantly mutated genes was performed between BTC-PCPD cohort and TCGA cohort of gastric cancer. We observed important differences between two cohorts. 13 significantly mutated genes reported by TCGA were covered by BTC-PCPD panel. Among them, only 6 genes were analyzed as significantly mutated genes by MutSigCV in BTC-PCPD cohort, including TP53 (59.09\%), PTEN (13.64\%), FBXW7 (2.60\%), CDH1 (13.64\%), SMAD4 (7.79\%) and $A P C$ (5.84\%) (Fig. 1c). Compared with TCGA result, 29 novel significantly mutated genes were identified in this study (Fig. 1c), and the top five most frequently mutated genes were AKAP9 (14.94\%), DRD2 (14.29\%), ATM (11.69\%), NOTCH2 (10.39\%) and LRP2 (10.39\%).

We first focused on DRD2 among the 29 novel significantly mutated genes. DRD2 gene is located in 11q23.2, encoding D2 subtype of the dopamine receptor. DRD2 was found to mutate at a higher frequency in this study $(14.29 \%)$ than in TCGA cohort (2.76\%) (Fig. 1b). Almost all mutation sites were located in 7 transmembrane receptor $\left(7 \mathrm{tm} \_1\right)$ functional region with dense distribution, and DRD2 p.H303P was the mutational hotspot (Fig. 2a). Dopamine (DA), a neurotransmitter, has been reported to play an important role in tumor progression [13]. Previous studies indicated prominence of DR signaling in human cancer and cancer progression. H Huang's study indicated dopamine D2 receptor suppresses invasion and migration of gastric cancer cells via inhibition of EGFR/AKT/MMP-13 pathway [14]. While, Mu's study reported that high expression of $D R D 2$ is correlated with poor prognosis of gastric cancer [15]. Dopamine D2 receptor was also reported to serve as biomarker of cancer stem cells for diverse malignancies. Dopamine D2-like receptor (D2DR) antagonists such as thioridazine could selectively target cancer stem cell, while having no effect on normal blood stem cells [16].

$L R P 2$ is located in 2q31.1, encoding low density lipoprotein-related protein 2 (megalin), which is critical for the reuptake of numerous ligands, including lipoproteins, sterols, vitamin-binding proteins, and hormones [17]. Unlike the case in DRD2, mutation sites were scattered in LRP2 (Fig. 2b). LRP2 is involved in cell uptake of vitamin D [18]. Megalin is acell surface protein abundantly present in the kidney that binds DBP (vitamin D binding protein) to mediate internalization of $25(\mathrm{OH}) \mathrm{D}$ into the cytosol [17]. LRP2 variants have previously been associated with prostate cancer [19] and pancreatic cancer risk [20]. Ge et al. first reported $L R P 2$ as a significantly mutated gene (7.7\%) in gastric cancer in 2017 [7]. But it is controversial whether vitamin D intake, serum vitamin D levels and vitamin $\mathrm{D}$ pathway were associated with gastric cancer $[21,22]$.

\section{Somatic copy number alterations (SCNAs) detection}

153 tumors were profiled for SCNAs of 22 autosomes (Fig. 3a). There were several significant peaks of copy number gain (Fig. 3b) including 7p11.2 (EGFR), 8q24.21 (MYC), 10q26.13 (FGFR2, MMP21), 12p12.1 (KRAS), 17q12 (ERBB2, CDK12, GRB7), 19q12 (CCNE1), and 20q13.2 (ZNF217). Figure 3c showed the top twenty SCNA genes according to frequency in samples. Among them, amplifications were detected in 13 genes, while deletions were found in 5 genes. Both amplification and deletion were identified in RYR1 and AKAP9 genes. Amplifications mainly targeted well-known oncogenes such as EGFR, ERBB2, KRAS, MYC, CCNE1, JAK2, FGFR2 et al. 12 gastric tumor tissues harbored CCNE1 amplifications (7.84\%), which was the most frequent genes with SCNA in this study, and with similar frequency (10.58\%) in TCGA cohort [4]. Amplification of CCNE1 is associated with
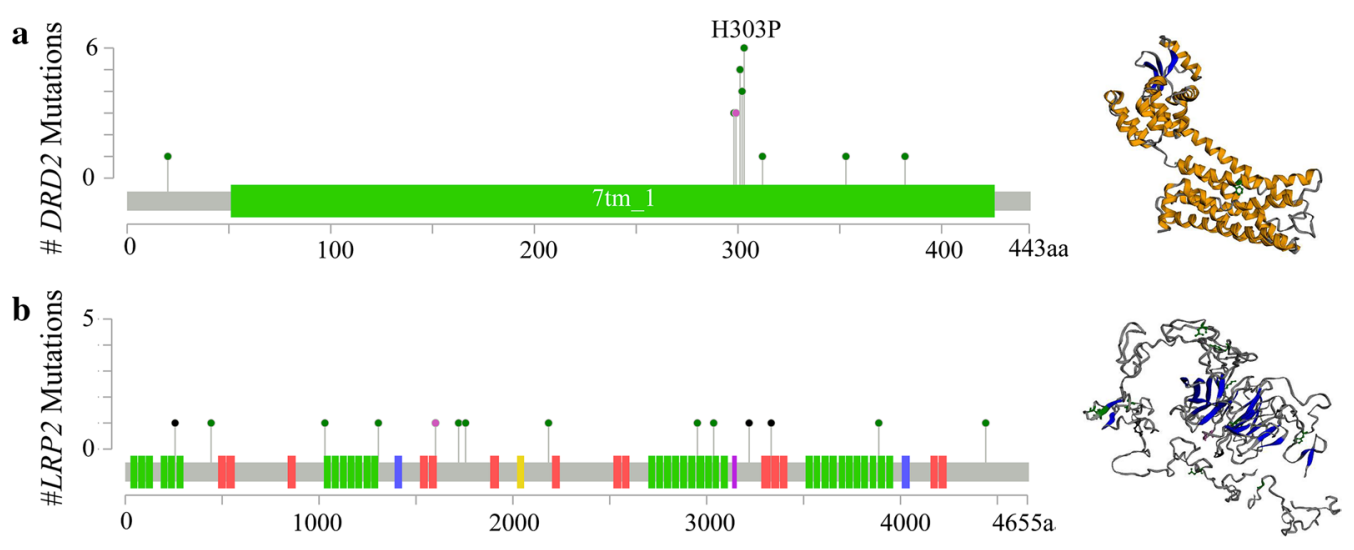

Fig. 2 The proportion of mutations and protein structure of $\mathbf{a} D R D 2$ and $L R P 2$ (b) 

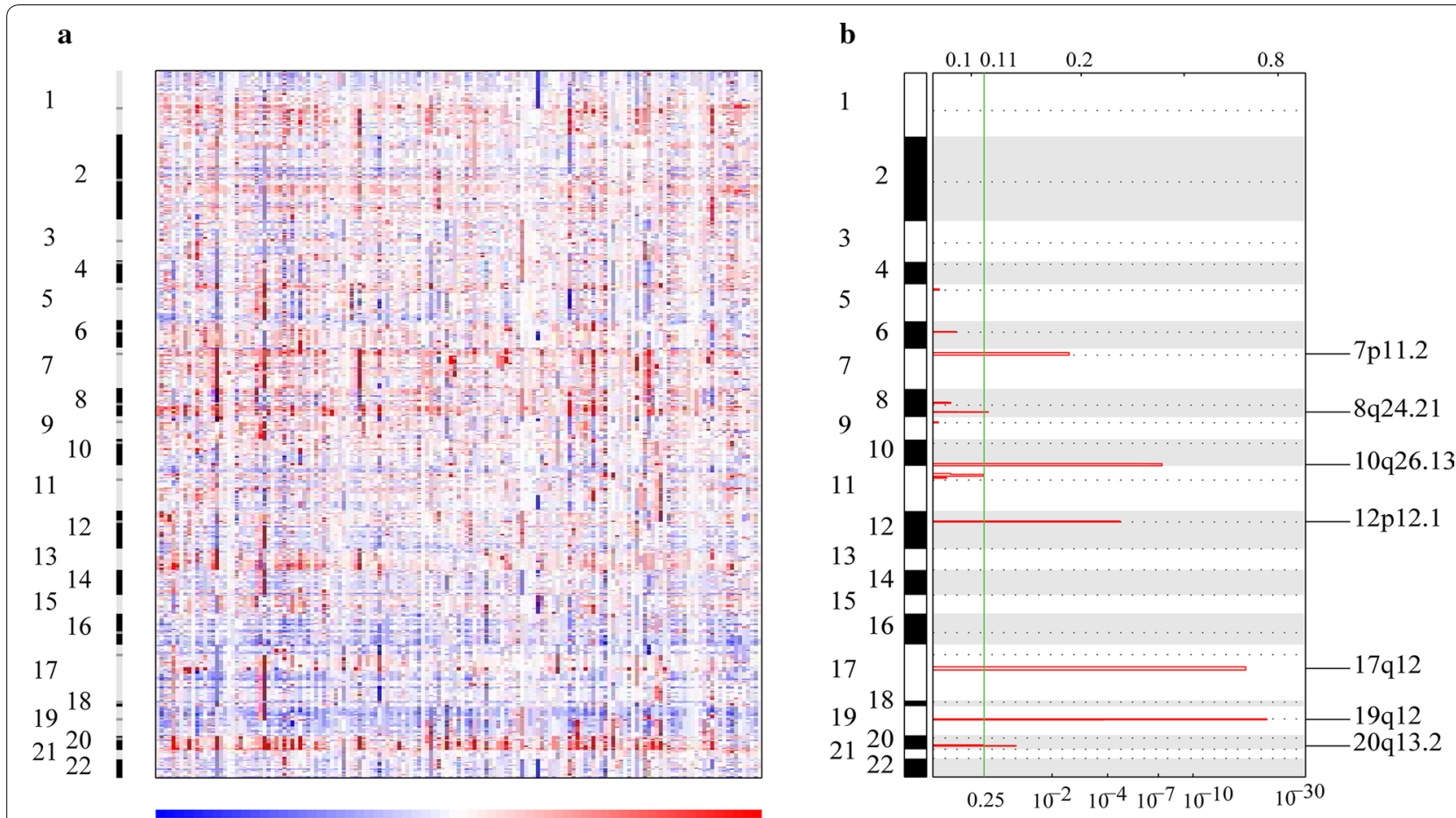

Loss

Gain

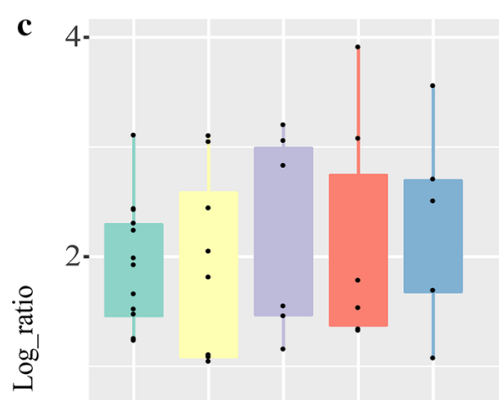

0 -
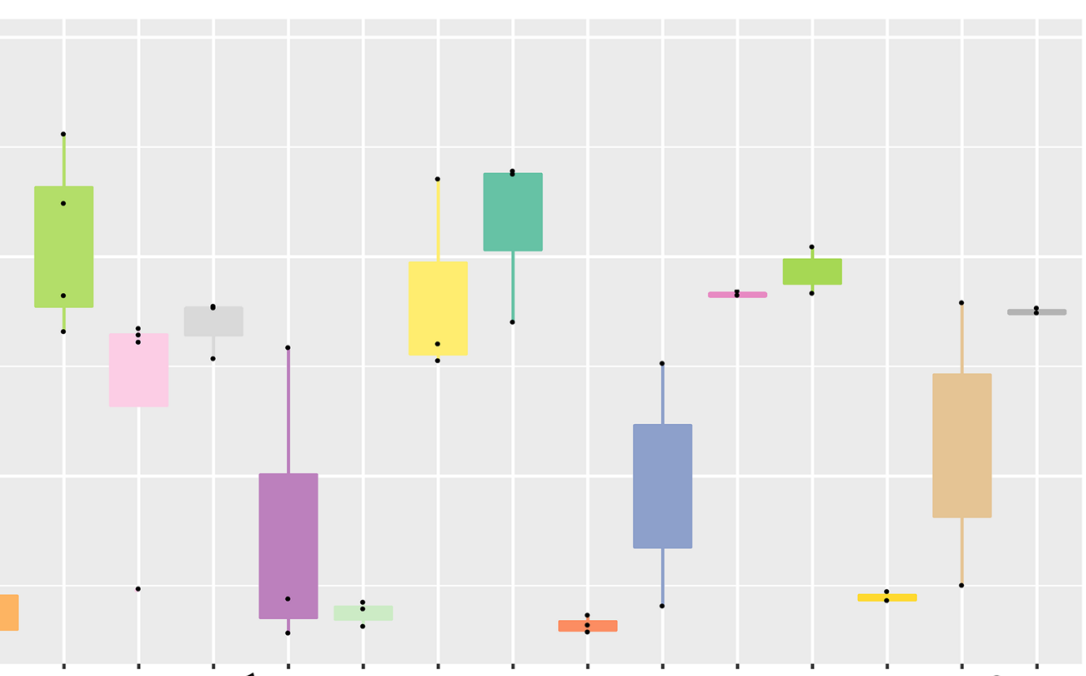

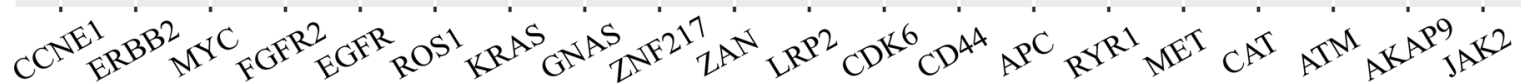

Fig. 3 Somatic copy number alterations (SCNAs). a SCNAs in tumors are plotted by chromosomal location (vertical axis) by GISTIC 2.0 analysis of the entire dataset. b Focal amplifications. Chromosomal locations of significantly recurring focal amplifications by false discovery rates using GISTIC 2.0. Annotated peaks have an FDR $<0.25$. No significantly recurring focal deletions were annotated. $\mathbf{c}$ The top 20 frequency genes

poor outcome in gastric [23], breast [24], and ovarian cancer $[25,26]$. ERBB2 amplification was detected in 8 gastric tumor tissues (5.23\%). Among 8 samples with ERBB2 amplifications, 6 samples (75\%) were positive by immunohistochemical (IHC) of erb-b2 receptor tyrosine kinase 2
(Additional file 4), which was the target of trastuzumab. Additionally, we also found amplification of CD44, which is a gastric stem cell marker [27].

We identified deletions in several genes including ROS1, ZAN, APC, LRP2, ATM et al. (Fig. 3c). ROS1 
encodes an orphan receptor tyrosine kinase related to anaplastic lymphoma kinase (ALK) [28]. It is well-known that ROS1 could be activated by chromosomal rearrangement in various human cancers such as non-small-cell lung cancer (NSCLC) [29]. ROS1 deletion was found in 4 samples in this study, while ROS1 amplification and deletion were reported in 2 samples each in TCGA cohort of 293 samples [4]. ROS1 amplifications and deletions were also reported to be heterogeneous in NSCLC, which had no impact on overall survival [10]. In this study, both $L R P 2$ and $A P C$ deletion were detected in three samples, and $A T M$ deletion was found in 2 samples, which were also reported deletions by TCGA.

\section{Enrichment of somatic mutated genes by GO and KEGG analysis}

To better understand the biological function of mutated genes, GO and KEGG enrichment analysis were performed. Altered signaling pathways included cell movement related pathways (adherens junction, focal adhesion and regulation of actin cytoskeleton), PI3K-Akt signaling, ErbB signaling, VEGF signaling, MAPK signaling, Ras signaling, p53 signaling, JAK-STAT pathway, and other well-known pathways. Figure 4 showed the top 15 pathways according to gene count and $\mathrm{p}$ value enriched by KEGG (Fig. 4a) and GO (Fig. 4b). In particular, we focused on alteration in PI3K-Akt signaling pathway, which is a key driver in carcinogenesis. As previous studies reported, PI3K signaling pathway was recurrently activated in gastric cancer [4], colorectal cancer [30], breast cancer [31] and other cancers [32, 33]. 93 genes with somatic mutations were enriched to PI3KAkt signaling pathway. $13.07 \%$ patients harbored mutations in PTEN gene, which is a tumor suppressor of the
PI3K-Akt pathway with eventual functional inactivation of this gene product. Involvement of several molecules of the PI3K-Akt pathways in GC carcinogenesis has eventually led to development of both single, as well as recently, dual inhibitors essential for molecular targeted therapy for GC, including pan-class I inhibitors, isoform specific PI3K inhibitors, Akt inhibitors, dual Akt/mTOR inhibitors [34]. GO enrichment result showed that most of functional categories were related with kinase activity (Fig. 4b), such as protein tyrosine kinase activity.

\section{Clinical actionability for targeted therapy}

To evaluate the clinical utility of prospective molecular profiling to guide treatment decisions, we used OncoKB (http://oncokb.org/) to group all mutations into various levels according to evidence of clinical actionability (Fig. 5a). Altogether, $38.31 \%$ of patients harbored at least one actionable alteration (Fig. 5b). We found a group of gene mutations as standard care biomarkers for an FDAapproved drug in another indication. $10.39 \%$ tumors harbored level_2B gene alterations (Fig. 5b) including In-frame shift of $K I T$, frameshift of $B R C A 2, \mathrm{SNVs}$ of $K I T, B R C A 1$ and $M E T$, and amplifications of ERBB2 and $M E T$ (Fig. 5c, d). Level_3B accounted for 6.49\% (Fig. 5b), including missense mutations ofERBB2, PIK3CA, $M A P 2 K 1$, In-frameshift of PIK3CA, and amplifications ofFGFR1 and MDM2 (Fig. 5c, d). Level_4 accounted for 21.43\% (Fig. 5b), including SNVs in PTEN, BRAF, KRAS, $A T M, C D K N 2 A$, amplification of EGFR and deletions of PTEN (Fig. 5c, d). Specifically, we found 22 actionable alterations of PTEN in 10 patients, which was the target of PI3K inhibitors AZD8186, as well as GSK2636771, and was classified as Level_4 (Fig. 5c).
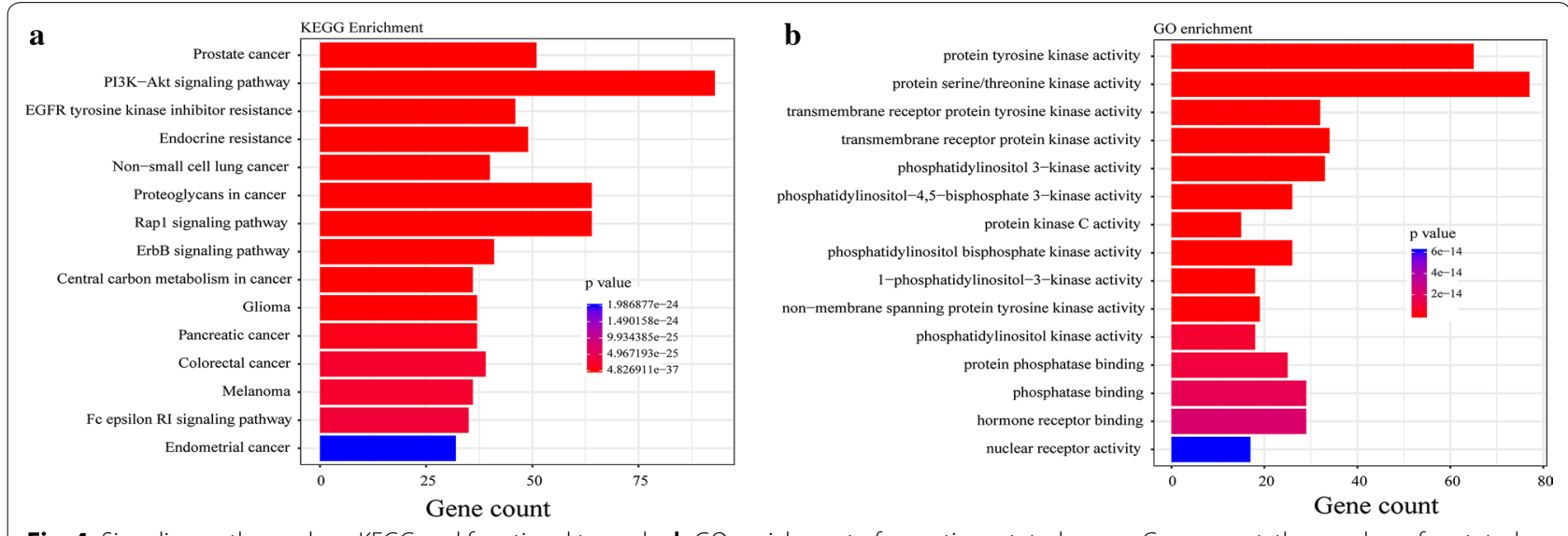

Fig. 4 Signaling pathways by a KEGG and functional terms by $\mathbf{b} \mathrm{GO}$ enrichment of somatic mutated genes. Gene count: the number of mutated genes enriched in this pathway or functional term 
a

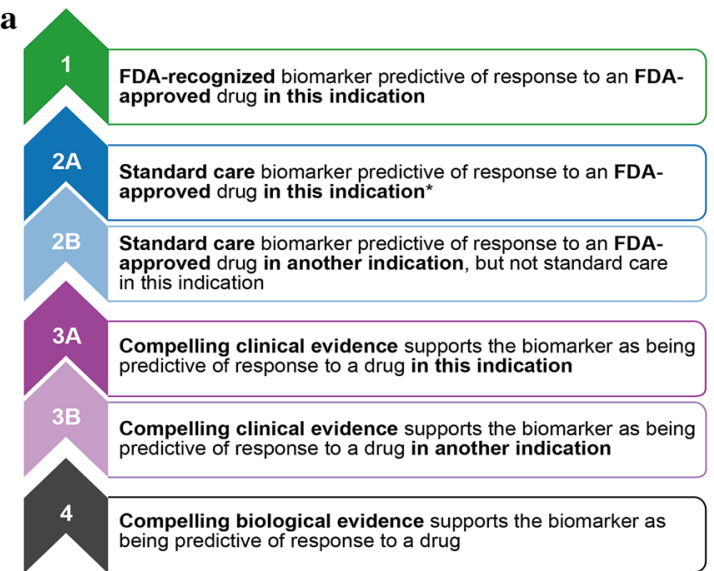

c

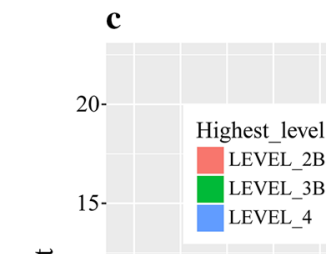

苛 10 -

5 -
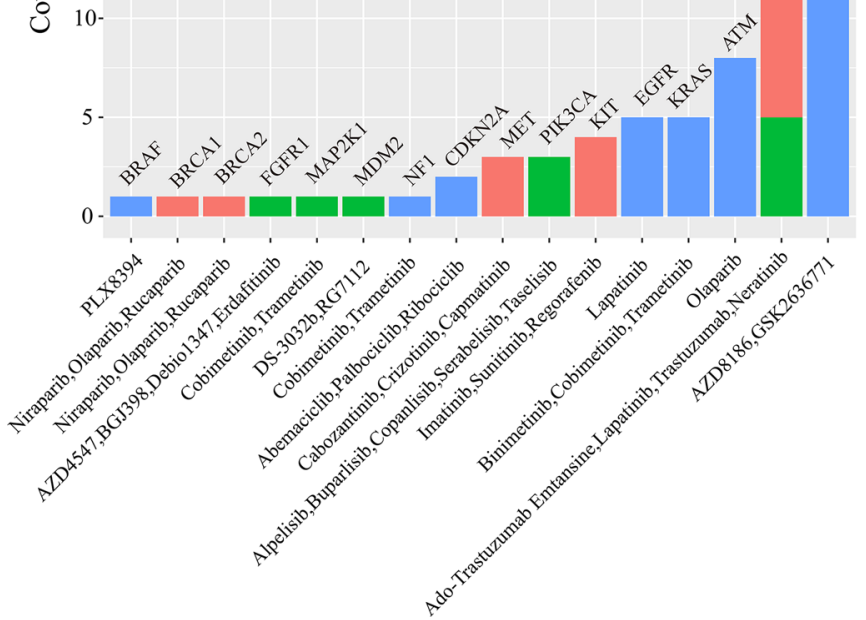

b

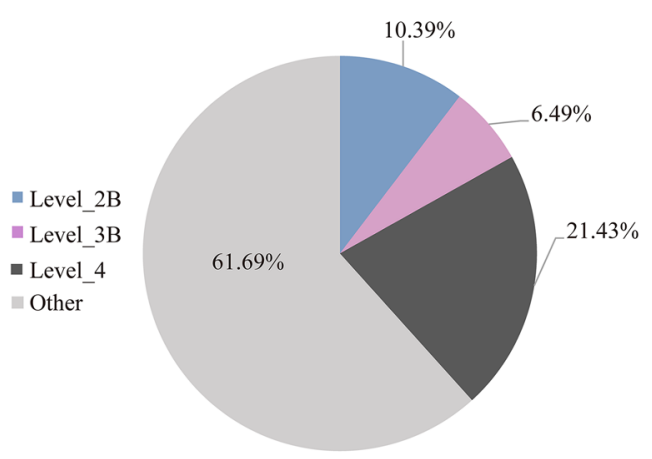

d

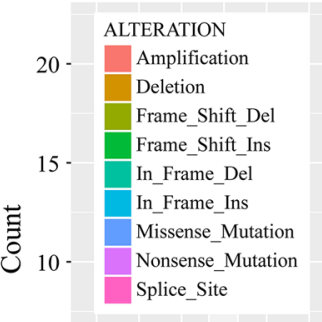

Fig. 5 Clinical actionability of somatic alterations revealed by BTC-PCPD. a Alterations were defined based on their clinical evidence according to OncoKB. b Samples were assigned to the highest level of actionable alterations. c Distribution of levels of actionable alterations. $\mathbf{d}$ Distribution of alteration types

\section{Tumor mutation burden of gastric cancer}

High tumor mutation burden (TMB) is an emerging biomarker of sensitivity to immune checkpoint inhibitors such as PD-1 and PD-L1 blockade immunotherapy. NGS panel is a reliable technology to analyze TMB, which was well reported by previous studies [35, 36]. In this study, we evaluated TMB of all 153 gastric tumors by BTC-PCPD panel (612 genes, $2.75 \mathrm{Mb}$ capture region). The median TMB of all samples was 5.801 (range 0.725-88.470) mutations/Mb (Fig. 6a). To the best of our knowledge, there is no uniform standard for high TMB yet. Currently, TMB levels could be divided into three groups based on the Foundation Medicine official reports: low (1-5 mutations/Mb), intermediate (6-19 mutations/Mb), and high ( $\geq 20$ mutations/Mb) [37], which was also applied in this study. We thus divided 4 patients $(2.61 \%)$ to high TMB, 63 patients $(41.18 \%)$ to intermediate TMB, 86 patients $(56.21 \%)$ to low TMB (Fig. 6a). Zehir's study using MSK-IMPACT panel indicated that the threshold for tumors with a high TMB was 

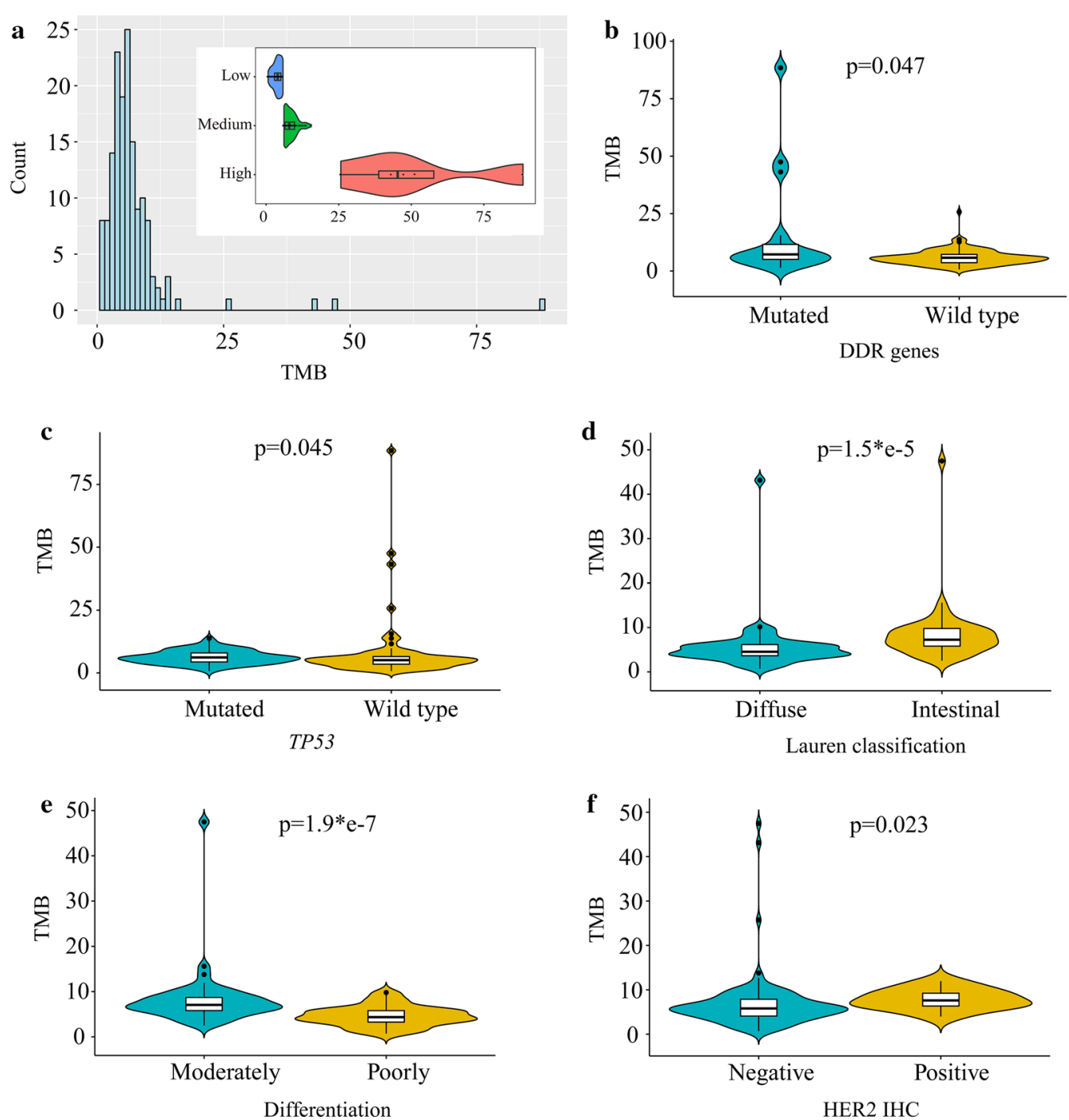

Fig. 6 Tumor mutation burden of gastric cancer. a The distribution of TMB across all tumors, using a threshold of low (1-5 mutations/Mb), intermediate (6-19 mutations/Mb), and high ( $\geq 20$ mutations/Mb). Non-parametric test of TMB according to DDR genes (b) and TP53 (c) genotype, Lauren classification (d), differentiation (e), and HER2 status (f)

13.8 mutations/Mb [6]. Accordingly, 5 tumors with TMB above 13.8 mutations/Mb was identified as high TMB in this study.

Mutations in DNA damage repair (DDR) genes occur as both germline polymorphisms and somatic mutations, including mismatch repair (MMR), base excision repair (BER) and homology-dependent recombination (HR), nucleotide excision repair (NER) and so on [38]. Defects in DNA replication, if not properly repaired, can lead to increased somatic mutation rate [35]. Twenty-seven genes within BTC-PCPD panel were previously identified as DDR related [38]. In this study, we analyzed deleterious germline mutations (potential pathogenic germline variants) and somatic mutations in DDR genes. Tumors with somatic mutations in DDR genes got higher TMB than wild-type tumors (p-value $=0.047$ ) (Fig. 6b). But we found no difference on TMB of DDR genes with germline mutations.

There were significant associations between TMB and TP53 genotype $(p=0.045$, Fig. $6 c)$, Lauren classification $(\mathrm{p}=1.5 \mathrm{e}-5$, Fig. $6 \mathrm{~d})$, differentiation $(\mathrm{p}=1.9 \mathrm{e}-7$, Fig. 6e), as well as HER2 IHC status ( $p=0.023$, Fig. 6f). TP53mutated tumors had higher median TMB, but lower mean TMB than TP53 wild-type tumors, and the similar phenomenon was found in TCGA dataset. In this study, all 5 high-TMB tumors ( $>13.8$ mutations $/ \mathrm{Mb}$ ) gathered in TP53 wild-type tumors. In TCGA dataset, we also found more high-TMB tumors with TP53 wild-type, but 
the difference was not significant $(\mathrm{p}=0.088)$ (Additional file 5). Intestinal type and moderately differentiated tumors got higher TMB than diffuse and poorly differentiated type. HER2 positive tumors had higher median TMB value, but all high-TMB tumors belonged to HER2 negative tumors.

\section{Discussion}

Gastric cancer is a genomically heterogeneous disease. Here, we characterized and provided extensive data describing tumor genomic mutation profile across 153 gastric cancer patients in the clinical setting. We assessed SNVs, InDels, SCNAs and TMB of tumors by BTC-PCPD panel, a target capture assay, which contributed to guiding the selection of genomically matched therapies for patients. Altogether, $38.31 \%$ of patients harbored at least one actionable alteration (Fig. 5) for treatment according to clinical evidence, which were classified into Level_2B, Level_3B, and Level_4. Unfortunately, no gene mutation was classified as Level_1 (FDA-recognized biomarker for an FDA-approved drug in the same indication). Although few patients with actionable alterations received genomically matched therapy because of medical, logistical and economic considerations, a group of patients still had choice to participate in clinical trials of matched targeted drugs, or try drugs in other indications.

We observed a wide range of TMB $(0.725-88.470$ mutations/Mb), and five patients were identified as high TMB (>13.8 mutations/Mb), with which patients may respond to immune-checkpoint inhibitors. It is hypothesized that highly mutated tumors are more likely to harbor neoantigens, which make them targets of activated immune cells. Increased mutation rate is well-characterized feature of human cancer. We found high TMB tumors tended to be enriched in tumors with wild-type TP53, somatic mutated DDR genes, and negative HER2. Previous studies reported that advanced urothelial carcinoma patients with DDR genes alterations could benefit from Platinumbased [39] and PD-1/PD-L1 blockade [40] therapy.

In this study, we identified 35 significantly mutated genes, as expected, the tumor suppressor gene TP53 is the top frequently mutated gene. We found that TP53 genotype was not only associated with clinical characteristic (gender and tumor location) but also tumor mutation burden (TMB). TP53 mutated tumors had higher median TMB than wild-type tumors. But interestingly, particular high TMB (TMB $>13.8$ mutations per $\mathrm{Mb}$ ) was enriched in TP53 wild type tumors. Loss of function mutations in TP53 are very common in various cancers and are a somatic marker of elevated mutation rate [41]. Instead, in lung adenocarcinoma, previous studies reported TP53 mutated more frequently in high TMB tumors (define: $>4.85$ mutations/Mb) [42], and had potential predictive value for response to PD-1 blockade immunotherapy. In addition, we found 29 novel significantly mutated genes which were not reported as significantly mutated genes in TCGA study.

\section{Conclusions}

Taken together, we developed a hybridization capturebased NGS panel (BTC-PCPD), drew a comprehensive mutational landscape of 153 gastric tumors, and demonstrated utility of massively parallel DNA sequencing of tumors to guide clinical management. There are limitations in our study. Survival analysis was not performed because of short time from patient enrollment. In the future, we will perform long term follow-up for these GC patients and recruit more new GC patients.

\section{Additional files}

\section{Additional file 1. Gene list of BTC-PCPD panel.}

Additional file $\mathbf{2}$. The proportion of mutations of 35 significantly mutated genes.

Additional file 3. Comparison of frequency of all mutated genes identified by this study between TCGA cohort and BTC-PCPD cohort.

Additional file 4. Immunostaining for the ERBB2 protein, $I \mathrm{HC}$ score $3+$. Additional file 5. Comparison of TMB level between TP53 mutated andwild type tumors in gastric cancer of TCGA dataset.

\section{Abbreviations}

GC: gastric cancer; NGS: next-generation sequencing; WES: whole-exome sequencing; BTC-PCPD: Biotecan PanCancer Panoramic Detection; FFPE: Formalin-Fixed and Paraffin-Embedded; TMB: tumor mutation burden; CNA: copy number variation; GO: gene ontology; KEGG: Kyoto Encyclopedia of Genes and Genomes; MSI: microsatellite instability; TCGA: The Cancer Genome Atlas.

\section{Acknowledgements \\ Not applicable.}

\section{Authors' contributions}

KY, MMN funded this study; HC, XSC, CQJ designed project and wrote the manuscript. DD, TH, JCY, ZML, XGH, ZRL, JSW, LS collected samples and clinical data. JMZ, LL, SXW performed experiments and analyzed data; KY, MMN, JMZ, PM revised the manuscript. All authors read and approved the final manuscript.

\section{Funding}

This work was supported by Scientific Research Project of Shanghai Health and Family Planning Committee (Grant No. 201640017), National Science and Technology Major Project (Grant No. 2017ZX09304030), and Major Projects of Special Development Funds in Zhangjiang National Independent Innovation Demonstration Zone, Shanghai (ZJ2017-ZD-012).

\section{Availability of data and materials}

Data sharing is applicable to this article.

\section{Ethics approval and consent to participate}

This study was approved by the Ethics Committee of Changhai Hospital and Shandong Provincial Hospital. Informed consent was obtained from individuals for the study. 


\section{Consent for publication}

Informed consent was obtained from all participants for publication.

\section{Competing interests}

The authors declare that they have no competing interests.

\begin{abstract}
Author details
1 Department of Gastrointestinal Surgery, Changhai Hospital, The Second Military Medical University, 168 Changhai Road, Yangpu District, Shanghai 200433, China. ${ }^{2}$ Department of Gastrointestinal Surgery, Shandong Provincial Hospital Affiliated to Shandong University, Jinan 250021, China. ${ }^{3}$ Zhangjiang Center for Translational Medicine, Shanghai Biotecan Pharmaceuticals Co., Ltd., 180 Zhangheng Road, Shanghai 201204, China. ${ }^{4}$ Shanghai Zhangjiang Institute of Medical Innovation, Shanghai 201204, China.
\end{abstract}

Received: 25 January 2019 Accepted: 29 May 2019 Published online: 04 June 2019

\section{References}

1. Torre LA, Bray F, Siegel RL, Ferlay J, Lortet-Tieulent J, Jemal A. Global cancer statistics, 2012. CA Cancer J Clin. 2015;65:87-108.

2. Chen W, Zheng R, Baade PD, Zhang S, Zeng H, Bray F, Jemal A, Yu XQ, He J. Cancer statistics in China, 2015. CA Cancer J Clin. 2016;66:115-32.

3. Lauren P. The two histological main types of gastric carcinoma: diffuse and so-called intestinal-type carcinoma. An attempt at a histo-clinical classification. Acta Pathol Microbiol Scand. 1965;64:31-49.

4. Cancer Genome Atlas Research N. Comprehensive molecular characterization of gastric adenocarcinoma. Nature. 2014;513:202-9.

5. Chen K, Yang D, Li X, Sun B, Song F, Cao W, Brat DJ, Gao Z, Li H, Liang $\mathrm{H}$, et al. Mutational landscape of gastric adenocarcinoma in Chinese: implications for prognosis and therapy. Proc Natl Acad Sci USA. 2015;112:1107-12.

6. Zehir A, Benayed R, Shah RH, Syed A, Middha S, Kim HR, Srinivasan P, Gao J, Chakravarty D, Devlin SM, et al. Mutational landscape of metastatic cancer revealed from prospective clinical sequencing of 10,000 patients. Nat Med. 2017;23:703-13.

7. Ge S, Li B, Li Y, Li Z, Liu Z, Chen Z, Wu J, Gao J, Shen L. Genomic alterations in advanced gastric cancer endoscopic biopsy samples using targeted next-generation sequencing. Am J Cancer Res. 2017;7:1540-53.

8. Xu Z, Huo X, Ye H, Tang C, Nandakumar V, Lou F, Zhang D, Dong H, Sun H, Jiang $S$, et al. Genetic mutation analysis of human gastric adenocarcinomas using ion torrent sequencing platform. PLoS ONE. 2014:9:e100442.

9. Kim HS, Lee H, Shin SJ, Beom SH, Jung M, Bae S, Lee EY, Park KH, Choi YY, Son T, et al. Complementary utility of targeted next-generation sequencing and immunohistochemistry panels as a screening platform to select targeted therapy for advanced gastric cancer. Oncotarget. 2017;8:38389-98

10. Clave S, Gimeno J, Munoz-Marmol AM, Vidal J, Reguart N, Carcereny E, Pijuan L, Menendez S, Taus A, Mate JL, et al. ROS1 copy number alterations are frequent in non-small cell lung cancer. Oncotarget. 2016:7:8019-28

11. Lawrence MS, Stojanov P, Polak P, Kryukov GV, Cibulskis K, Sivachenko A, Carter SL, Stewart C, Mermel CH, Roberts SA, et al. Mutational heterogeneity in cancer and the search for new cancer-associated genes. Nature. 2013:499:214-8.

12. Yu G, Wang LG, Han Y, He QY. clusterProfiler: an R package for comparing biological themes among gene clusters. OMICS. 2012;16:284-7.

13. Basu S, Dasgupta PS. Role of dopamine in malignant tumor growth. Endocrine. 2000;12:237-41.

14. Huang H, Wu K, Ma J, Du Y, Cao C, Nie Y. Dopamine D2 receptor suppresses gastric cancer cell invasion and migration via inhibition of EGFR/ AKT/MMP-13 pathway. Int Immunopharmacol. 2016:39:113-20.

15. Mu J, Huang W, Tan Z, Li M, Zhang L, Ding Q, Wu X, Lu J, Liu Y, Dong Q, $\mathrm{Xu} \mathrm{H}$. Dopamine receptor D2 is correlated with gastric cancer prognosis. Oncol Lett. 2017;13:1223-7.

16. Sachlos E, Risueno RM, Laronde S, Shapovalova Z, Lee JH, Russell J, Malig M, McNicol JD, Fiebig-Comyn A, Graham M, et al. Identification of drugs including a dopamine receptor antagonist that selectively target cancer stem cells. Cell. 2012;149:1284-97.
17. Christensen El, Birn H. Megalin and cubilin: multifunctional endocytic receptors. Nat Rev Mol Cell Biol. 2002;3:256-66.

18. Andreassen TK. The role of plasma-binding proteins in the cellular uptake of lipophilic vitamins and steroids. Horm Metab Res. 2006;38:279-90.

19. Holt SK, Karyadi DM, Kwon EM, Stanford JL, Nelson PS, Ostrander EA. Association of megalin genetic polymorphisms with prostate cancer risk and prognosis. Clin Cancer Res. 2008;14:3823-31.

20. Anderson LN, Cotterchio M, Knight JA, Borgida A, Gallinger S, Cleary SP. Genetic variants in vitamin d pathway genes and risk of pancreas cancer; results from a population-based case-control study in ontario. Canada. PLOS ONE. 2013:8:e66768.

21. Khayatzadeh S, Feizi A, Saneei P, Esmaillzadeh A. Vitamin D intake, serum Vitamin D levels, and risk of gastric cancer: A systematic review and metaanalysis. J Res Med Sci. 2015;20:790-6.

22. Eom SY, Yim DH, Kim DH, Yun HY, Song YJ, Youn SJ, Hyun T, Park JS, Kim BS, Kim YD, Kim H. Dietary vitamin D intake and vitamin D related genetic polymorphisms are not associated with gastric cancer in a hospital-based case-control study in Korea. J Biomed Res. 2018;32:257-63.

23. Wang D, Zhu H, Guo M, Fan X, Hu S, Yan K, Sun J, Wang J, Li M, Xiao H, Liu Z. Expression and prognostic value of cell-cycle-associated genes in gastric adenocarcinoma. BMC Gastroenterol. 2018;18:81.

24. Hunt KK, Karakas C, Ha MJ, Biernacka A, Yi M, Sahin AA, Adjapong O, Hortobagyi GN, Bondy M, Thompson P, et al. Cytoplasmic cyclin E predicts recurrence in patients with breast cancer. Clin Cancer Res. 2017:23:2991-3002

25. Etemadmoghadam D, Au-Yeung G, Wall M, Mitchell C, Kansara M, Loehrer E, Batzios C, George J, Ftouni S, Weir BA, et al. Resistance to CDK2 inhibitors is associated with selection of polyploid cells in CCNE1-amplified ovarian cancer. Clin Cancer Res. 2013;19:5960-71.

26. Ayhan A, Kuhn E, Wu RC, Ogawa H, Bahadirli-Talbott A, Mao TL, Sugimura $H$, Shih IM, Wang TL. CCNE1 copy-number gain and overexpression identify ovarian clear cell carcinoma with a poor prognosis. Mod Pathol. 2017;30:297-303.

27. Liu G, Neumeister M, Reichensperger J, Yang RD. Therapeutic potential of human adipose stem cells in a cancer stem cell-like gastric cancer cell model. Int J Oncol. 2013:43:1301-9.

28. Acquaviva J, Wong R, Charest A. The multifaceted roles of the receptor tyrosine kinase ROS in development and cancer. Biochim Biophys Acta. 2009;1795:37-52.

29. Shaw AT, Ou SH, Bang YJ, Camidge DR, Solomon BJ, Salgia R, Riely GJ, Varella-Garcia M, Shapiro Gl, Costa DB, et al. Crizotinib in ROS1-rearranged non-small-cell lung cancer. N Engl J Med. 2014;371:1963-71.

30. Cancer Genome Atlas N. Comprehensive molecular characterization of human colon and rectal cancer. Nature. 2012;487:330-7.

31. Cancer Genome Atlas N. Comprehensive molecular portraits of human breast tumours. Nature. 2012:490:61-70.

32. Schulze K, Imbeaud S, Letouze E, Alexandrov LB, Calderaro J, Rebouissou S, Couchy G, Meiller C, Shinde J, Soysouvanh F, et al. Exome sequencing of hepatocellular carcinomas identifies new mutational signatures and potential therapeutic targets. Nat Genet. 2015;47:505-11.

33. Cancer Genome Atlas Research N. Comprehensive molecular profiling of lung adenocarcinoma. Nature. 2014;511:543-50.

34. Singh SS, Yap WN, Arfuso F, Kar S, Wang C, Cai W, Dharmarajan AM, Sethi G, Kumar AP. Targeting the PI3K/Akt signaling pathway in gastric carcinoma: a reality for personalized medicine? World J Gastroenterol. 2015;21:12261-73.

35. Chalmers ZR, Connelly CF, Fabrizio D, Gay L, Ali SM, Ennis R, Schrock A, Campbell B, Shlien A, Chmielecki J, et al. Analysis of 100,000 human cancer genomes reveals the landscape of tumor mutational burden. Genome Med. 2017:9:34

36. Wang Z, Duan J, Cai S, Han M, Dong H, Zhao J, Zhu B, Wang S, Zhuo M, Sun J, et al. Assessment of blood tumor mutational burden as a potential biomarker for immunotherapy in patients with non-small cell lung cancer with use of a next-generation sequencing cancer gene panel. JAMA Oncol. 2019. https://doi.org/10.1001/jamaoncol.2018.7098.

37. Goodman AM, Kato S, Bazhenova L, Patel SP, Frampton GM, Miller V, Stephens PJ, Daniels GA, Kurzrock R. Tumor mutational burden as an independent predictor of response to immunotherapy in diverse cancers. Mol Cancer Ther. 2017;16:2598-608.

38. Knijnenburg TA, Wang L, Zimmermann MT, Chambwe N, Gao GF, Cherniack AD, Fan H, Shen H, Way GP, Greene CS, et al. Genomic and molecular 
landscape of DNA damage repair deficiency across the cancer genome atlas. Cell Rep. 2018;23(239-254):e236.

39. Teo MY, Bambury RM, Zabor EC, Jordan E, Al-Ahmadie H, Boyd ME, Bouvier N, Mullane SA, Cha EK, Roper N, et al. DNA damage response and repair gene alterations are associated with improved survival in patients with platinum-treated advanced urothelial carcinoma. Clin Cancer Res. 2017;23:3610-8

40. Teo MY, Seier K, Ostrovnaya I, Regazzi AM, Kania BE, Moran MM, Cipolla CK, Bluth MJ, Chaim J, Al-Ahmadie H, et al. Alterations in DNA damage response and repair genes as potential marker of clinical benefit from PD-1/PD-L1 blockade in advanced urothelial cancers. J Clin Oncol. 2018;36:1685-94.

41. Petitjean A, Mathe E, Kato S, Ishioka C, Tavtigian SV, Hainaut P, Olivier M. Impact of mutant p53 functional properties on TP53 mutation patterns and tumor phenotype: lessons from recent developments in the IARC TP53 database. Hum Mutat. 2007;28:622-9.

42. Offin M, Rizvi H, Tenet M, Ni A, Sanchez-Vega F, Li BT, Drilon A, Kris MG, Rudin CM, Schultz N, et al. Tumor mutation burden and efficacy of EGFRtyrosine kinase inhibitors in patients with EGFR-mutant lung cancers. Clin Cancer Res. 2018. https://doi.org/10.1158/1078-0432.CCR-18-1102.

\section{Publisher's Note}

Springer Nature remains neutral with regard to jurisdictional claims in published maps and institutional affiliations.
Ready to submit your research? Choose BMC and benefit from:

- fast, convenient online submission

- thorough peer review by experienced researchers in your field

- rapid publication on acceptance

- support for research data, including large and complex data types

- gold Open Access which fosters wider collaboration and increased citations

- maximum visibility for your research: over $100 \mathrm{M}$ website views per year

At BMC, research is always in progress.

Learn more biomedcentral.com/submissions 